\title{
目 \\ MONOTONE INVARIANTS AND EMBEDDINGS OF STATISTICAL MANIFOLDS
}

\author{
HÔNG VÂN LÊ \\ MAX-PLANCK-INSTITUTE FOR MATHEMATICS IN SCIENCES \\ INSELSTRASSE 22-26 \\ D-04103 LEIPZIG \\ EMAI: HVLE@MIS.MPG.DE
}

\begin{abstract}
In this note we prove certain necessary and sufficient conditions for the existence of an embedding of statistical manifolds. In particular, we prove that any compact smooth $\left(C^{1}\right.$ resp.) statistical manifold can be embedded into the space of probability measures on a finite set. As a result, we get an answer to the Lauritzen question on a realization of smooth $\left(C^{1}\right.$ resp.) statistical manifolds as statistical models.
\end{abstract}

MSC: 53C42, 60D05, 65H20.

Keywords: Fisher metric, Chentsov-Amari connections, statistical manifolds, statistical models.

\section{INTRODUCTION}

A statistical model is a family $M$ of probability measures on a measurable space $\Omega$. There are two natural geometrical structures on any statistical model equipped with a differentiable manifold structure. They are the Fisher tensor and the Chentsov-Amari tensor.

The Fisher tensor was given by Fisher in 1925 as an information characterization of a statistical model. Rao [Rao(1945)] proposed to consider this tensor as a Riemannian metric on the manifold of probability distributions. This Fisher metric has been systematically studied in [Chentsov1972], [M-C 1990], [A-N2000] and others [Lauritzen1987], [Rao1987], [Ay2002], [Jost2005], ect. in the field of geometric aspects of statistics and information theory.

Chentsov [Chentsov1972] and Amari [Amari1997] independently also discovered a natural structure on statistical models, namely a 1-parameter family of invariant connections, which includes the Levi-Civita connection of the Fisher metric. This family of invariant connections is defined by a 3 -symmetric tensor $T$ together with the Levi-Civita connection of the Fisher metric.

\footnotetext{
1appeared in "Advances in Deterministic and Stochastic Analysis", World Scientific Hackensack, NJ, 2007, 231-254.
} 
Motivated by the question how much we can describe a statistical model via their Fisher metric and Chentsov-Amari tensor $T$, in 1987 Lauritzen proposed to call a Riemannian manifold $(M, g)$ with a 3 -symmetric tensor $T$ a statistical manifold. Since two 3 -symmetric tensors $T$ and $k \cdot T, k \neq 0$, define the same family of Chentsov-Amari connections, we shall say that two statistical manifolds $(M, g, T)$ and $(M, g, k T)$ are conformal equivalent.

A natural and important question in the mathematical statistics is to understand, if a given family $M$ of probability distributions can be considered as a subfamily of another given one $N$. In the language of statistical manifolds, this question can be formulated as a problem of isostatistical embedding of a statistical manifold $(M, g, T)$ into another one $\left(N, g^{\prime}, T^{\prime}\right)$. Here we say that an immersion $f:(M, g, T) \rightarrow(\bar{M}, \bar{g}, \bar{T})$ is called isostatistical, if $f^{*}(\bar{g})=g$ and $f^{*}(\bar{T})=T$.

We shall see in section 2 that the problem of the existence of an isostatistical embedding includes also the Lauritzen question in 1987, if any statistical manifold is a statistical model. It also concerns the following important problem posed by Amari in 1997, if any finite dimensional statistical model can be embedded into the space $\operatorname{Cap}^{N}$ of probability distributions of the sample space $\Omega^{N}$ of $N$ elementary events for some finite $N$.

We shall construct a class of $C^{0}$ (and $C^{1}$ ) monotone invariants of statistical manifolds, which present obstructions to embedding of a given $C^{k}$ statistical manifold $M$ into another one $N^{n}$. Here a $C^{k}$ statistical manifold $(M, g, T)$ is a smooth differentiable manifold with $C^{k}$ sections $g \in S^{2} T^{*} M$ and $T \in S^{3} T^{*} M$. These invariants measure certain relations between the metric tensor $g$ and the 3-symmetric tensor $T$. In particular, using these invariants we show that no statistical manifold which is conformal equivalent to the space $\mathrm{Cap}^{N}$ can be embedded into the product of $m$ copies of the normal Gaussian manifolds for any $N>3$ and any finite $m$. In the Main Theorem (section 5$)$ we prove that any compact smooth $\left(C^{1}\right.$ resp.) statistical manifold $M^{m}$ can be isostatistically embedded to a the space $C a p^{N}$ for some $N$ big enough.

As a consequence we also get a new proof of Matumoto theorem on the existence of the contrast function for a compact statistical manifold (see 2.8).

Acknowledgement. I am thankful to Jürgen Jost and Nihat Ay for their introduction to the field of information geometry and helpful discussions.

\section{Statistical MODELS AND STATisticAl MANiFoldS.}

In this section we recall the definitions of the Fisher metric and the Chentsov-Amari connections on statistical models. We introduce the notion of a weak Fisher metric and a weak potential function. At the end of the section we discuss the problem, if a given statistical manifold is a statistical model. Most of the facts in this section can be found in [A-N2000]. 
Suppose that $M$ is a statistical model - a family of probability measures on a space $\Omega$. We assume throughout this note that $M$ and $\Omega$ are differentiable manifolds, and $\Omega$ is equipped with a fixed Borel measure $d \omega$. We also write

$$
p(x, \omega)=p(x, \omega) d \omega,
$$

where $p(x, \omega)$ in LHS of $(2.1)$ is a Borel measure in $M$ and $p(x, \omega)$ in the RHS of (2.1) is a non negative (density) function on $M \times \Omega$ which satisfies

$$
\int_{\Omega} p(x, \omega) d \omega=1 \forall x \in M .
$$

The Fisher metric $g^{F}(x)$ is defined on $M$ as follows. For any $V, W \in T_{x} M$ we put

$$
g^{F}(V, W)_{x}=\int_{\Omega}\left(\partial_{V} \ln p(x, \omega)\right)\left(\partial_{W} \ln p(x, \omega)\right) p(x, \omega) .
$$

The function under integral in (2.2) is well defined, if

$$
p(x, \omega)>0,
$$

Denote by $\operatorname{Cap}(\Omega)$ the space of all probability measures on $\Omega$. Clearly we can consider the density function $p(x, \omega)$ as a mapping $M \rightarrow \operatorname{Cap}(\Omega)$. Thus we shall call a function $p(x, \omega)$ a probability potential of the metric $g_{F}$, if $p(x, \omega)$ satisfies (2.1.a), (2.1.b), (2.2). (It is known that for a given Riemannian metric $g_{F}$ on a smooth manifold $M$ there exist many probability potentials $f(x, \omega)$ for $g_{F}$, even if we fix the space $(\Omega, d \omega)$.)

Some time it is useful to consider functions $p(x, \omega)$ which satisfy $(2.2)$ and (2.1.b) but not necessary (2.1.a). In this case, the Riemannian metric $g^{F}$ will be called weak Fisher metric, and the function $p(x, \omega)$ will be called a weak probability potential of $g^{F}$.

2.3. Example of a weak Fisher metric: the standard Euclidean metric $g^{0}$ on the positive quadrant $\mathbb{R}_{+}^{N}\left(x_{i}>0\right)$. It is straightforward to check that $g_{0}$ admits a weak probability potential $\left\{p_{i}(x)=\frac{1}{4} x_{i}^{2}, i=\overline{1, N}\right.$. $\}$ Here $\Omega=\Omega^{N}$ - the sample space of $N$ elementary events.

2.4. The Fisher metric on the space $\left(\mathrm{Cap}^{N}\right)_{+}$of all positive probability distributions on $\Omega^{N}$ (see also [A-N2000], [Jost2005], [Chentsov1972]). By definition we have

$$
C a p_{+}^{N}:=\left\{\left(p_{1}, \cdots, p_{N}\right) \mid p_{i}>0 \text { for } i=\overline{1, N} \& \sum p_{i}=1\right\} .
$$

We define the embedding map

$$
\begin{gathered}
f: \operatorname{Cap}_{+}^{N} \rightarrow S^{N-1}(2), \\
\left(p_{1}, \cdots, p_{N}\right) \mapsto\left(q_{1}=2 \sqrt{p_{1}}, \cdots, q_{N}=2 \sqrt{p_{N}}\right) .
\end{gathered}
$$

It is easy to see that the Fisher metric in the new coordinates $\left(q_{i}\right)$ is the standard metric of constant positive curvature on the sphere $S^{N-1}(2)$. 
2.5. Divergence potential (see [A-N2000], [Rao(1987)].) A function $\rho$ on $M \times M$ with the following property

$$
\rho(x, y) g^{F} e 0 \text { with equality iff } x=y
$$

is called a divergence function. A divergence function $\rho$ is called a divergence potential for a metric $g$ on $M$, if

$$
g(X, Y)_{x}=\operatorname{Hess}(\rho)\left(i_{1}(X), i_{1}(Y)\right) .
$$

where

$$
T_{(x, x)}(M, M)=\left(T_{x} M, 0\right) \oplus\left(0, T_{x} M\right)=\left(i_{1}\left(T_{x} M\right)\right) \oplus\left(i_{2}\left(T_{x} M\right)\right) .
$$

An example of a divergence potential for a Fisher metric is the Jensen function $J_{H}^{\lambda, \mu}(x, y)$ of the entropy function $H(x)$ on $M$, or a Kullback relative entropy function $K(x, y)$ on $M \times M$.

2.6. Chentsov-Amari connections. Let $p(x, \omega)$ be a probability potential for a Riemannian metric $g$. We define a symmetric 3 -tensor $T$ on $M$ as follows

$$
T(X, Y, Z)=\int\left(\partial_{X} \ln p(x, \omega)\right)\left(\partial_{Y} \ln p(x, \omega)\right)\left(\partial_{Z} \ln p(x, \omega)\right) p(x, \omega) .
$$

We denote by $\nabla^{F}$ the Levi-Civita connection of the (weak) Fisher metric $g^{F}$. We define

$$
<\nabla_{X}^{t} Y, Z>:=<\nabla_{X}^{F} Y, Z>+t \cdot T(X, Y, Z) .
$$

The connections $\nabla^{t}$ are called the Chentsov-Amari connections.

2.6.3. Remark. ([A-N2000], [Matsumoto1993]) Any divergence function $\rho(x, y)$ on $M \times M$ defines a tensor $T$ on $M$ via the following formula

$T(X, Y, Z)_{x}=-\partial_{i_{2}(Z)} \operatorname{Hess}(\rho)\left(i_{1}(X), i_{1}(Y)\right)_{(x, x)}+\partial_{i_{1}(Z)} H \operatorname{ess}(\rho)\left(i_{2}(X), i_{2}(Y)\right)_{(x, x)}$.

If $g$ and $T$ are defined by the same divergence function $\rho(x, y)$, we shall call $\rho(x, y)$ a divergence potential for the statistical manifold $(M, g, T)$. It is a known fact that the Kullback relative entropy function is a divergence potential for the associated statistical model.

\subsection{Statistical submanifolds.}

A submanifold $N$ in a statistical manifold $(M, g, T)$ with the induced Riemannian metric $g_{\mid N}$ and induced tensor $T_{\mid N}$ is called statistical submanifold of $(M, g, T)$. Clearly, if $f(x, \omega)$ is a (weak) probability potential for $(M, g, T)$, then its restriction to any submanifold $N \subset M$ is a (weak) probability potential of the induced statistical structure.

2.8. Statistical models and statistical manifolds. Since any probability function $p(x, \omega)$ defines a map $M \rightarrow \operatorname{Cap}(\Omega)$, we shall say that a statistical manifold $(M, g, T)$ is a statistical model, if there probability potential $p(x, \omega)$ for $g$ and $T$. By the remark in 2.7, we get that a statistical submanifold of a statistical model is also a statistical model. Furthermore, 
if a statistical manifold $(M, g, T)$ is a statistical model, then it must admit a divergence potential. Hence we obtain the following

2.8.1 Theorem. (cf. [Matumoto1993] ) For any compact statistical manifold $(M, g, T)$ there exists a divergence potential $\rho$ for $g$ and for $T$.

Note that Matumoto's theorem does not requires the compactness of $(M, g, T)$.

\section{EMBEDdings OF LinEAR STATISTICAL SPACES.}

An Euclidean space $\left(\mathbb{R}^{n}, g^{0}\right)$ equipped with a 3 -symmetric tensor $T$ will be called a linear statistical spaces. We observe that the equivalence class of linear statistical spaces coincides with the orbit space of 3-symmetric tensors $T$ under the action of the orthogonal group $O(n)$. In this section we discuss certain invariants of these orbits and we show several necessary and sufficient conditions for the existence of embedding of one linear statistical space into another linear statistical space by studying these invariants. A class of our necessary conditions consists of monotone invariants $\lambda$, i.e. we assign to any linear statistical space $\left(\mathbb{R}^{n}, g^{0}, T\right)$ a number $\lambda\left(\mathbb{R}^{n}, g^{0}, T\right)$ such that, if $\left(\mathbb{R}^{n}, g^{0}, T\right)$ is a statistical submanifold of $\left(\mathbb{R}^{m}, g^{0}, T^{\prime}\right)$, then we have

$$
\lambda\left(\mathbb{R}^{n}, g^{0}, T\right) \leq \lambda\left(\mathbb{R}^{m}, g^{0}, T^{\prime}\right) .
$$

Since a tangent space of a statistical manifold is a linear statistical manifold, these invariants play important role in the problem of isostatistical immersion.

3.1. Trace type of a symmetric 3-tensor. Let us denote by $\mathcal{R}^{n}$ the subspace in $S^{3}\left(\mathbb{R}^{n}\right)$ consisting of the following 3-symmetric tensors

$$
T^{v}(x, y, z)=<v, x><y, z>+<v, y><x, z>+<v, z><x, y>,
$$

where $v \in \mathbb{R}^{n}$. Using the standard representation theory (see e.g. [ON1988]) we have the decomposition

$$
S^{3}\left(\mathbb{R}^{n}\right)=\mathcal{R}\left(3 \pi_{1}\right) \oplus \mathcal{R}^{n} .
$$

The component $\mathcal{R}^{n}$ is defined by taking the trace of $T$

$$
\left.\operatorname{Tr}: S^{3}\left(\mathbb{R}^{n}\right)^{*} \rightarrow\left(\mathbb{R}^{n}\right)^{*}, \operatorname{Tr}(T)(v):=\operatorname{Tr}(v\rfloor T\right) .
$$

Clearly $\operatorname{Tr}$ is an $S O(n)$-equivariant map with nonzero image. Using the identity $\operatorname{Tr}\left(T^{v}\right)=(n+2) v^{*}$, we get

3.3. Lemma. We have

$$
\pi_{2}(T)=\frac{1}{n+2} T^{T r(T)} .
$$

In view of Lemma 3.3 we shall call any tensor $T \in \mathcal{R}^{n}$ of trace type. 
We note that

$$
\operatorname{dim} S^{3}\left(\mathbb{R}^{n}\right)=C_{n}^{3}+2 C_{n}^{2}+n=\frac{n(n+1)(n+2)}{6} .
$$

Thus the dimension of the quotient $S^{3}\left(\mathbb{R}^{n}\right) / S O(n)$ is at least $C_{n}^{3}+C_{n}^{2}+n$. A direct computation shows that the dimension of the orbit $S O(n)\left(\left[\sum_{i=1}^{n} a_{i} v_{i}^{3}\right]\right)$ is $C_{n}^{2}=\operatorname{dim} S O(n)$, if $\Pi a_{i} \neq 0$. Here $\left\{v_{i}\right\}$ is an orthonormal basis in $\mathbb{R}^{n}$. Hence the dimension of $S^{3}\left(\mathbb{R}^{n}\right) / O(n)=C_{n}^{3}+C_{n}^{2}+n$. This dimension is exactly the number of all complete invariants of pairs consisting of a positive definite bilinear form $g$ and a 3 -symmetric tensor $T$.

Since the dimension of $G_{k}\left(\mathbb{R}^{n}\right)=k(n-k)$, it follows that generically it is impossible to embed a linear statistical space $\left(R^{k}, g^{0}, T\right)$ into a given statistical linear space $\left(R^{n}, g^{0}, T\right)$, unless $k(n-k) g^{F} e C_{k}^{3}+C_{k}^{2}+k$. Clearly the dimension condition is not sufficient as the following proposition shows.

3.5. Proposition. A linear statistical space $\left(\mathbb{R}^{k}, g^{0}, T\right)$ can be embedded into a linear statistical space $\left(\mathbb{R}^{N}, g^{0}, T^{v}\right)$, if and only if $N g^{F}$ ek and $T$ is also a trace type: $T=T^{w}$ with $|w| \leq|v|$.

Proof. The necessary condition follows from the fact that the restriction of $T^{v}$ to $\mathbb{R}^{k}$ equals $T^{\bar{v}}$, where $\bar{v}$ is the orthogonal projection of $v$ to $\mathbb{R}^{k}$. Conversely, if $|w| \leq|v|$ we can find an orthogonal transformation, such that $w$ equals the orthogonal projection of $v$ on $\mathbb{R}^{k}$.

3.6. Commasses as monotone invariants. Since the metric $g$ extends canonically on the space $S^{3}\left(\mathbb{R}^{n}\right)$, we can define the absolute norm

$$
\|T\|:=\sqrt{<T, T>}
$$

Now we define comasses of a 3 -symmetric tensor $T$ as follows

$$
\begin{gathered}
\mathcal{M}^{3}(T):=\max _{|x|=1,|y|=1,|z|=1} T(x, y, z), \\
\mathcal{M}^{2}(T):=\max _{|x|=1,|y|=1} T(x, y, y), \\
\mathcal{M}^{1}(T):=\max _{|x|=1} T(x, x, x) .
\end{gathered}
$$

Clearly we have

$$
0 \leq \mathcal{M}^{1}(T) \leq \mathcal{M}^{2}(T) \leq \mathcal{M}^{3}(T) \leq\|T\|
$$

3.7. Proposition. The comasses $\mathcal{M}^{i}, i \in[1,3]$, are nonnegative linear monotone invariants, which vanish if and only if $T=0$.

Proof. Clearly $\mathcal{M}^{i}(T) \geq 0$ for $i=1,2,3$. Now we are going to show that $\mathcal{M}^{1}$ vanishes at $T$ only if $T=0$. Observe that $\mathcal{M}^{1}=0$ if and only if $T(x, x, x)=0$ for all $x \in \mathbb{R}^{n}$. Writing $T$ in coordinate expression $T(x, y, z)=$ $\sum a_{i j k} x^{i} y^{j} z^{k}$, we note that $T(x, x, x)=0$ if and only if $T=0$, since $T$ is symmetric. 
Next we shall show that $\mathcal{M}^{i}(T)$ is a linear monotone invariant for $i=$ $1,2,3$. Assume that $e$ is a linear embedding $\left(\mathbb{R}^{n}, g, T\right)$ into $\left(\mathbb{R}^{m}, \bar{g}, \bar{T}\right)$. Then $T$ is a restriction of the 3 -symmetric tensor $\bar{T}$. Hence we have

$$
\mathcal{M}^{i}(T) \leq \mathcal{M}^{i}(\bar{T}), \text { for } i=1,2,3 .
$$

This implies that $\mathcal{M}^{i}$ are linear monotone invariants.

Now for a space $\left(\mathbb{R}^{n}, g^{0}, T\right)$ and for $1 \leq k \leq n$ we put

$$
\lambda_{k}(T):=\min _{\mathbb{R}^{k} \subset \mathbb{R}^{n}} \mathcal{M}^{1}\left(T_{\mid \mathbb{R}^{k}}\right) .
$$

We can easily check that if $\bar{T}$ is a restriction of $T$ to a subspace $\mathbb{R}^{m} \subset \mathbb{R}^{n}$, then

$$
\lambda_{k}(\bar{T}) \geq \lambda_{k}(T) \geq 0 \text { for all } k \leq m .
$$

Thus $-\lambda_{k}(T)$ is a monotone invariant of linear statistical manifolds. These invariants are related by the following inequalities

$$
\mathcal{M}^{1}(T)=\lambda_{n}(T) \geq \lambda_{n-1}(T) \cdots \geq \lambda_{2}(T) \geq \lambda_{1}(T)=0 .
$$

The last equality follows from the fact, that the function $T(x, x, x)$ is anti-symmetric on $S^{n-1}(|x|=1) \subset \mathbb{R}^{n}$ and $S^{n-1}$ is connected. We observe that if $T$ is of trace type, then $\lambda_{n-1}(T)=\cdots=\lambda_{1}(T)=0$.

We are going to give a lower bound of the monotone invariant $\lambda_{n-1}$ of a linear statistical space of certain type. The equality $\lambda_{n-1}\left(\mathbb{R}^{n}, g^{0}, T\right) g^{F} e A$ means that no hyperplane with the norm $\mathcal{M}^{1}$ strictly less than $A$ can be embedded in $\left(\mathbb{R}^{n-1}, g^{0}, T\right)$.

3.8. Lemma. a) Let $T=\sum_{i=1}^{n}\left(N-\varepsilon_{i}\right)\left(x^{i}\right)^{3}$ be a 3-symmetric tensor on $\mathbb{R}^{n}$ with $n \geq 4, N \geq 4$ and $\left|\varepsilon_{i}\right| \leq 1 / 4$. Then we have

$$
\lambda_{n-1}(T) \geq \frac{N}{\sqrt{10}}-1 / 4
$$

b) Let $T=N \sum_{i=1}^{n}\left(x^{i}\right)^{3}$, and $H$ be a hyperplane in $\mathbb{R}^{n}$ which is orthogonal to $(k n, 1,1, \cdots, 1)$, and let $n \geq 5, k \geq 3$. Then we have

$$
\lambda_{n-2}\left(T_{\mid H}\right) \geq \frac{N}{5}-1 \text {. }
$$

c) Let $x=\left((1-\varepsilon), \frac{1}{k n}, \cdots, \frac{1}{k n}\right) \in S^{n}(1) \subset \mathbb{R}^{n+1}$, where $n \geq 4, k \geq n$. We denote by $H$ the tangential plane $T_{x} S^{n}$, and by $T^{0}$ the following 3-symmetric tensor on $\mathbb{R}_{+}^{n+1}$ :

$$
T_{i j k}^{0}\left(x_{1}, \cdots x_{N}\right)=\delta_{i j k} \frac{2}{x_{i}}
$$

Then we have

$$
\lambda_{n-1}\left(T_{\mid H}^{0}\right) \geq \frac{k n}{5}-1 .
$$

3.8.2. Remark. The tensor $T^{0}$ in (3.8.1) defines on $\left(\mathbb{R}^{n}, g^{0}\right)$ a statistical structure with a weak probability potential $\left\{\frac{1}{4} x_{i}^{2}, i=1, n\right\}$, see also 2.3 . 
Proof of Lemma 3.8. The reader shall see that a proof of Lemma 3.8 can be done in the same scheme of the proof of Sublemma 5.10. Therefore we do not repeat this argument here.

3.8.3. Remark. Lemma 3.8. a holds also for $n=3$ but not for $n=2$, Lemma 3.8.b holds also for $n=4$, but not for $n=3$, and Lemma 3.8.c holds also for $n=3$ but not for $n=2$.

There are also several obvious monotone invariants of $T$.

$$
A^{1}(T):=\max _{|x|=|y|=|z|=1,<x, y>=<y, z>=<z, x>=0} T(x, y, z)
$$

is well-defined for $n \geq 3$.

$$
A^{2}(T):=\max _{|x|=|y|=1,<x, y>=0} T(x, y, y),
$$

is well-defined for $n \geq 2$. We can check that

$$
\text { ker } A^{1}=\mathcal{R}^{n} \text {. }
$$

On the other hand we have

$$
\operatorname{ker} A^{2} \subset \mathcal{R}\left(3 \pi_{1}\right)
$$

Thus $A^{1}$ and $A^{2}$ are different invariants.

3.9. Lemma. Let $\pi_{1}$ be the first component of $T$ in decomposition (3.2). Then $\|T\|_{1}:=\left\|\pi_{1}(T)\right\|$ is a monotone invariant of $T$.

Proof. Let $\mathbb{R}^{k}$ be a subspace of $\mathbb{R}^{n}$. We denote by $\pi_{k}^{n} T$ the restriction of $T$ to $\mathbb{R}^{k}$. Clearly

$$
\pi_{k}^{n}(T)=\pi_{k}^{n}\left(\pi_{1} T\right)+\pi_{k}^{n}\left(\pi_{2} T\right) .
$$

We have noticed in Proposition 3.5 that the restriction of the trace form $\pi_{2} T$ to any subspace is also a trace form. Thus $\pi_{k}^{n}\left(\pi_{2}\right)$ is an element in $\mathcal{R}^{k} \subset S^{3}\left(\mathbb{R}^{k}\right)$. Hence we have

$$
\pi_{1}\left(\pi_{k}^{n} T\right)=\pi_{1}\left(\pi_{k}^{n}\left(\pi_{1} T\right)\right) .
$$

Since all the projections $\pi_{1}, \pi_{k}^{n}$ decrease the norm $\|$.$\| , we get$

$$
\left\|\pi_{k}^{n} T\right\|_{1}=\left\|\pi_{1}\left(\pi_{k}^{n} T\right)\right\|=\left\|\pi_{1}\left(\pi_{k}^{n}\left(\pi_{1} T\right)\right)\right\| \leq\left\|\pi_{1}(T)\right\|=\|T\|_{1} .
$$

3.10. Proposition. A statistical line $\left(\mathbb{R}, g^{0}, T\right)$ can be embedded into $\left(\mathbb{R}^{N}, g^{0}, T^{\prime}\right)$, if and only if $\mathcal{M}^{1}(T) \leq \mathcal{M}^{1}\left(T^{\prime}\right)$.

Proof. It suffices to show that we can embed $\left(\mathbb{R}, g^{0}, T\right)$ into $\left(\mathbb{R}^{N}, g^{0}, T^{\prime}\right)$, if we have $\mathcal{M}^{1}(T) \leq \mathcal{M}^{1}\left(T^{\prime}\right)$. We note that $T^{\prime}(v, v, v)$ defines an antisymmetric function on the sphere $S^{N-1}(|v|=1) \subset \mathbb{R}^{N}$. Thus there is a point $v \in S^{N-1}$ such that $T^{\prime}(v, v, v)=\mathcal{M}^{1}(T)$. Clearly the line $v \otimes \mathbb{R}$ defines the required embedding. 
Let us consider the embedding problem for 2-dimensional linear statistical spaces. It is easy to see that

$$
S^{3}\left(\mathbb{R}^{2}\right)=\mathbb{R}^{2} \oplus \mathbb{R}^{2}
$$

Thus the quotient $S^{3}\left(\mathbb{R}^{2}\right) / S O(2)$ equals $\left(\mathbb{R}^{2} \oplus \mathbb{R}^{2}\right) / S^{1}$. Geometrically there are several ways to see this. In the first way we denote components of $T \in S^{3}\left(\mathbb{R}^{2}\right)$ via $T_{111}, T_{112}, T_{122}, T_{222}$.

3.11. Lemma. There exists an oriented orthonormal basic in $\mathbb{R}^{2}$ such that $T_{111}=\mathcal{M}^{1}(T)>0, T_{112}=0$ for all non-vanishing $T$. These numbers $\left(T_{111}, T_{122}, T_{222}\right)$ are called canonical coordinates of $T$. Two tensors $T$ and $T^{\prime}$ are equivalent, if and only if they have the same canonical coordinates.

Proof. We choose an oriented orthonormal basis $\left(v_{1}, v_{2}\right)$ by taking as $v_{1}$ a point on $S^{1}(|x|=1)$, where the function $T(x, x, x)$ reaches the maximum. The first variation formula shows that in this case $T_{112}=0$. This shows the existence of the canonical coordinates. Clearly, if two tensors have the same canonical coordinates, then they are equivalent. Next, if two tensors $T$ and $T^{\prime}$ are equivalent, then their norms $\mathcal{M}^{1}$ are the same. We need to take care the case, when there are several points $x$ at which $T(x, x, x)$ reaches the maximum. In any case, they have the same first coordinates. Next we note that

$$
\begin{gathered}
<\operatorname{Tr}(T), \operatorname{Tr}(T)>=\left(T_{111}+T_{122}\right)^{2}+T_{222}^{2}, \\
\|T\|^{2}=T_{111}^{2}+T_{122}^{2}+T_{222}^{2} .
\end{gathered}
$$

Thus if two tensors are equivalent and have the same first coordinates, they must have the same third coordinate $T_{122}$, and this third coordinate is uniquely defined up to sign. The condition on the orientation tells us that the sign must be + . This proves the second statement.

3.12. Proposition. We can always embed the 2-dimensional statistical space $\left(\mathbb{R}^{2}, g^{0}, 0\right)$ into any linear statistical space $\left(\mathbb{R}^{n}, g^{0}, T\right)$, if $n \geq 7$.

Proof. It suffices to prove for $n=7$. We denote by $\mathcal{O}(T)$ the set of of all unit vectors $v \in S^{6}$ such that $T(v, v, v)=0$. Clearly $\mathcal{O}(T)$ is a set of dimension 5 in $S^{6}$. Since $T$ is anti-symmetric, there exists a connected component $\mathcal{O}^{0}(T)$ of $\mathcal{O}(T)$ which is invariant under the anti-symmetry involution. Now we consider the following function $f$ on $\mathcal{O}^{0}(T)$. For each $v \in \mathcal{O}^{0}(T)$ we denote by $A^{v}$ the bilinear symmetric 2 -form on the space $T_{x} \mathcal{O}^{0}(T)$ considered as a subspace in $\mathbb{R}^{n}$ :

$$
A^{v}(y, z)=T(v, y, z) .
$$

Then we define $f(v)$ equal to $\operatorname{det}\left(A^{v}\right)$. Since $\mathcal{O}(T)$ has dimension 5 , the function $f(v)$ is anti-symmetric on $\mathcal{O}^{0}(T)$. Hence the set $\mathcal{O}_{0}^{0}(T)$ of all $v \in \mathcal{O}^{0}(T)$ with $f(v)=0$ has dimension 4 and it contains a connected component which is also invariant under the anti-symmetric involution. For the simplicity we denote this connected component also by $\mathcal{O}_{0}^{0}(T)$. Now we consider the following two possible cases. 
Case 1. We assume that there is a point $v \in \mathcal{O}_{0}^{0}(T)$ such that the nullity of $A^{v}$ is at least 2. Then there are two linear independent vectors $y, z \in T_{v}$ such that the restriction of $A^{v}$ on the plane $\mathbb{R}^{2}(y, z)$ vanishes. Since the set $\mathcal{O}^{0}(T)$ is connected and anti-symmetric and of co dimension 1 in $S^{n-1}$, the plane $\mathbb{R}(y, z)$ has a non-empty intersection with $\mathcal{O}^{0}(T)$ at a point $w$. Then the restriction of $T$ on the plane $\mathbb{R}^{2}(v, w)$ is vanished, because

$$
\begin{gathered}
T(v, v, v)=T(w, w, w)=0 \\
T(v, w, w)=0\left(\text { since } A^{v}(w, w)=0\right), \\
T(v, v, w)=0\left(\text { since } w \in T_{v} \mathcal{O}^{0}(T)\right) .
\end{gathered}
$$

Case 2. We assume that the nullity of $A^{v}$ on $\mathcal{O}_{0}^{0}(T)$ is constantly 1 . Using the anti-symmetric property of $A^{v}$ we conclude that the restriction of $A^{v}$ to the plane $\mathbb{R}^{4}(v)$ which is orthogonal to the kernel of $A^{v}$ has index constantly 2 . Thus there exists a vector $z$ which is orthogonal to the kernel $y$ of $A^{v}$ such that $A^{v}(z, z)=0$. Clearly the restriction of $A^{v}$ to the plane $\mathbb{R}^{2}(y, z)$ vanishes. Now we can repeat the argument in the case 1 to get a vector $w$ such that the restriction of $T$ to $\mathbb{R}^{2}(v, w)$ vanishes.

3.13. Theorem. a) Any statistical space $\left(\mathbb{R}^{n}, g^{0}, T\right)$ can be embedded in the statistical space $\left(\mathbb{R}^{n(n+1)}, g^{0}, T^{\prime}=2\|T\| \sum_{i=1}^{N(n)} x_{i}^{3}\right)$, where $x_{i}$ are the canonical Euclidean coordinates on $\mathbb{R}^{n(n+1)}$.

b) The trivial space $\left(\mathbb{R}^{n}, g^{0}, 0\right)$ can be embedded into $\left(\mathbb{R}^{2 n}, g^{0}, \sum_{i=1}^{2 n}\left(d x^{i}\right)^{3}\right)$ for all $n$.

Proof. a) We prove by induction. The statement for $n=1$ follows from Proposition 3.8. Suppose that the statement is valid for all $n \leq k$.

3.14. Lemma. Suppose that $T \in S^{3}\left(\mathbb{R}^{k+1}\right)$. Then there are orthonormal coordinates $x_{1}, \cdots, x_{k}$ such that

$$
T=x_{1} \sum_{i=1}^{k+1} a_{i} x_{i}^{2}+\sum_{1<i, j, k} a_{i j k} x_{i} x_{j} x_{k} .
$$

Proof of Lemma 3.14. We choose $v_{1}$ as the unit vector in $S^{k} \subset \mathbb{R}^{k+1}$, on which the function $T(v, v, v)$ reaches the maximum on the unit sphere $S^{k}$. The first variation formula shows that $T\left(v_{1}, v_{1}, w\right)=0$ for all $w$ which is orthogonal to $v_{1}$. We denote by $\mathbb{R}^{k}$ the orthogonal complement to $\mathbb{R} \cdot v_{1}$. Now we consider a bilinear symmetric form $A$ on $\mathbb{R}^{k}$ defined as follows

$$
A(x, y)=S\left(v_{1}, x, y\right) .
$$

There is an orthonormal basis on $\mathbb{R}^{k}$, where we can write $A(x, y)=\sum_{i=2}^{k+1} a_{i} x_{i}^{2}$. Clearly in this orthonormal basis we can write $T$ in the form in (3.14.1).

Continuation of the proof of Theorem 3.13.a We shall show explicitly that that any statistical space $\left(\mathbb{R}^{2}, g^{0}, T=a_{2} x_{1}\left(x_{2}\right)^{2}\right)$ can be embedded in 
$\left(\mathbb{R}^{4}, g^{0}, \sum_{i=1}^{4}\left(y_{i}\right)^{3}\right)$, if $0 \leq\left|a_{2}\right| \leq 1 / 2$. We put

$$
\begin{gathered}
L\left(v_{1}\right):= \pm\left(\frac{1}{2}, \frac{1}{2},-\frac{1}{2},-\frac{1}{2}\right) \\
L\left(v_{2}\right):=\left(\sqrt{\frac{1+2 a_{2}}{2}},-\sqrt{\frac{1+2 a_{2}}{2}}, \sqrt{\frac{1-2 a_{2}}{2}},-\sqrt{\frac{1-2 a_{2}}{2}}\right) .
\end{gathered}
$$

Here we take the sign + in (3.16.1), if $a_{2}>0$, and we take the sign - , if $a_{2}<0$. Clearly, $L$ defines the required embedding $\mathbb{R}^{2} \rightarrow \mathbb{R}^{4}$.

This together with Proposition 3.8 and the induction assumption completes the proof of Theorem 3.13. a.

Proof of Theorem 3.13. $b$. We decompose the embedding $f:\left(\mathbb{R}^{n}, g^{0}, 0\right)$ to $\left(\mathbb{R}^{2 n}, g^{0}, \sum_{i=1}^{2 n}\left(x^{i}\right)^{3}\right)$ as follows

$$
f\left(x_{1}, \cdots, x_{n}\right)=\left(f^{1}\left(x_{1}\right), \cdots, f^{n}\left(x_{n}\right)\right)
$$

where $f^{i}$ embeds the line $\left(\mathbb{R},\left(d x^{i}\right)^{2}, 0\right)$ into $\left(\mathbb{R}^{2},\left(d x^{2 i-1}\right)^{2}+\left(d x^{2 i}\right)^{2},\left(d x^{2 i-1}\right)^{3}+\right.$ $\left.\left(d x^{2 i}\right)^{3}\right)$. Clearly, $f$ is the required embedding.

\section{Monotone invariants And obstructions to EMBEDdings of STATISTICAL MANIFOLDS}

Let $K(M, e)$ denote the category of statistical manifolds $M$ with morphisms being embeddings. Functors of this category are called monotone invariants of statistical manifolds. Clearly any monotone invariant is an invariant of statistical manifolds.

4.1. Examples. There are many monotone invariants which arise from our analysis in section 3 .

a) Trace type of a statistical manifold. A statistical manifold $(M, g, T)$ will be called of trace type, if for all $x \in M$ the form $T(x)$ is of trace type (see 3.1.) It follows from Proposition 3.5 that any statistical submanifold of a statistical manifold of trace type is also of trace type. Thus the trace type is a monotone invariant. In particular we cannot embed the statistical space $\mathrm{Cap}^{N}$ and the normal Gaussian space into any statistical space of trace type. On the other hand, unlike the linear case, we cannot embed a statistical manifold of trace type into another one of trace type, even if the norm condition is satisfied. For example, if the trace form is closed (or exact), then the trace form of its submanifolds is also closed (resp. exact). Hence within a class of statistical manifolds of trace type we get a new monotone invariants which can be expressed via the closedness and the cohomology class of the corresponding trace form.

b) Decomposability of a statistical manifold. We note that the class of 3 -symmetric tensors of trace form is a subclass of all decomposable tensors $T^{3}$ which are a symmetric product of 1 -forms and symmetric 2 -forms. Any statistical submanifold of a statistical manifold with a decomposable 
tensor $T$ has also the (induced) decomposable tensor. Thus the decomposability is also a monotone invariant. The Gaussian normal 2-dimensional manifold is an example of decomposable type but not of trace type.

c) Rank and comass. We define for any statistical manifold $(M, g, T)$ the following number

$$
\begin{aligned}
\operatorname{rank}(T) & =\sup \operatorname{rank}(T(x)) \\
\|T\|_{0} & =\sup _{x \in M}\|T(x)\| . \\
\mathcal{M}^{1}(T)_{0} & =\sup _{x \in M} \mathcal{M}^{1}(T(x)) . \\
\|T\|_{1,0} & =\sup _{x \in M}\|T(x)\|_{1} .
\end{aligned}
$$

Clearly these four numbers are monotone invariants of statistical manifolds.

We recall that the normal Gaussian statistical manifold is the two dimensional statistical model which is upper half of the plane $\mathbb{R}^{2}(\mu, \sigma)$ with the potential

here $x \in \mathbb{R}$.

$$
p(\mu, \sigma)(x)=\frac{1}{\sqrt{2 \pi} \sigma} \exp \left(\frac{-(x-\mu)^{2}}{2 \sigma^{2}}\right),
$$

4.2. Proposition. Any statistical manifold which is conformal equivalent to the space $\mathrm{Cap}^{N}$ cannot be embedded into the direct product of $m$ copies of the normal Gaussian statistical manifold 2.3.3.a for any $N \geq 3$ and finite $m$.

Proof. It is easy to check that $\mathcal{M}^{1}\left(\operatorname{Cap}^{N}\right)=\infty$. Thus any statistical manifold which is conformal equivalent to $\mathrm{Cap}^{N}$ has also the infinite invariant $\mathcal{M}^{1}$. On the other hand, we compute easily that the norm $\mathcal{M}^{1}$ of the Gaussian normal manifold, as well as the norm $\mathcal{M}^{1}$ of a direct product of its finite copies, is finite. Namely the norm $\mathcal{M}^{1}(\mu, \sigma)$ is $\sqrt{2}$ for all $(\mu, \sigma)$.

4.3. Diameters of statistical manifolds. For a positive number $\rho>0$ and a statistical manifold $(M, g, T)$ we set

$d_{\rho}(M, g, T):=\sup \left\{l \in R^{+} \cup \infty \mid \exists\right.$ an immersion of $\left([0, l], d x^{2}, \rho(d x)^{3}\right)$ to $\left.(M, g, T).\right\}$

We shall call $d_{\rho}(M, g, T)$ the diameter with weight $\rho$ of $(M, g, T)$. Clearly $d_{\rho}$ are monotone invariants for all $\rho$.

To estimate the diameter with weight $\rho$ of a given statistical manifold $(M, g, T)$ we can proceed as follows. For each point $x \in M$ we denote by $D_{\rho}(x)$ the set of all unit tangential vector $v \in T_{x} M$ such that $T(v, v, v)=\rho$. We denote by $D_{\rho}^{i}(x)$ the connected components of $D_{\rho}^{i}(x)$. We say that a unite vector $v$ in $T_{x} M$ is $\rho$-characteristic with weight $c(x)$, if there exists $i$ such that we have

$$
c(x)=\min _{w \in D_{\rho}^{i}(x)}<v, w>>0 .
$$


We shall say that a point $x \in M$ is $\rho$-regular, if there is an open neighborhood $U_{\varepsilon}(x) \subset M$ such that $D_{\rho}\left(U_{\varepsilon}\right)=U_{\varepsilon} \times D_{\rho}(x)$. It is easy to see that the set of all $\rho$-regular points is open and dense in $M$ for any given $\rho$.

4.4. Proposition. The diameter $d_{\rho}$ of $\left(M^{m}, g, T\right)$ is infinite, if $m \geq 3$ and there exists a number $\varepsilon>0$ such that one of the following 2 conditions holds:

a)There exists a $(\rho+\varepsilon)$-regular point $x \in M$ such that the convex hull $\operatorname{Cov}\left(D_{\rho+\varepsilon}^{i}(x)\right)$ of one of connected components $D_{\rho+\varepsilon}^{i}(x)$ contains the origin point $0 \in T_{x} M^{m}$ as it interior point.

b) $\left(M^{m}, g, T\right)$ has a complete Riemannian submanifold $(N, \bar{g})$ such that there exists a smooth section $x \mapsto\left(D_{\rho+\varepsilon}(x) \cap T N\right)$ over $N$.

Proof. The statement under the first condition a) is based on the fundamental Lemma of the convex integration technique of Gromov. Namely Gromov proved that [2.4.1.A, Gromov(1986)], if the convex hull of some path connected subset $A_{0} \subset \mathbb{R}^{q}$ contains a small neighborhood of the origin, then there exists a map $f: S^{1} \rightarrow \mathbb{R}^{q}$ whose derivative sends $S^{1}$ into $A_{0}$.

4.5. Lemma. Under the condition in Proposition 4.4.1 there exists a small neighborhood $U_{\delta}(x)$ in $M$ and an embedded oriented curve $S^{1} \subset U_{\delta}(x)$ such that for all point $s(t) \in S^{1}$ we have $\mathcal{M}^{1}\left(T_{s(t)} S^{1}\right) \geq \rho+(\varepsilon / 2)$.

Proof of Lemma 4.5. We denote by Exp the exponential map $T_{x} M^{m} \rightarrow$ $M^{m}$ and by DExp the differential of this exponential map restricted to $S^{m-1} \times T_{x} M^{m} \subset T\left(T_{x} M^{m}\right)$. Here $S^{m-1}$ is the unit sphere in $T_{x} M^{m}$. The space $T_{x} M^{m}$ is a linear statistical space, so we denote by $\mathcal{M}_{x}^{1}$ the induced norm-function on $S^{m-1} \times T_{x} M^{m}$ as follows:

$$
\mathcal{M}_{x}^{1}(l)=T_{x}(l, l, l) .
$$

Since $D E x p$ is a continuous function, whose restriction to $S^{m-1} \times 0$ is the identity, there exists a ball $B(0, \delta)$ with center in $0 \in T_{x} M$ such that

$$
\left.\mathcal{M}^{1}(D \operatorname{Exp}(l))-\mathcal{M}_{x}^{1}(l)\right)<\varepsilon / 4
$$

for all $l \in S^{m-1} \times B(\delta) \subset T\left(T_{x} M^{m}\right)$. We can assume that $\delta$ is so small such that DExp is a homeomorphism on $S^{m-1} \times B(0, \delta)$.

Now we apply the above mentioned Gromov Lemma [2.4.1.A, Gr1986] to get a oriented curve $S^{1}(t)$ in the linear space $T_{x} M$ such that

$$
T\left(\frac{(\partial / \partial t) S^{1}(t)}{\left|(\partial / \partial t) S^{1}(t)\right|}\right)=\rho+\varepsilon
$$

for all $t$. Next we observe that for all $\alpha>0$ the curve $\alpha \cdot S^{1}(t)$ has the same norm as $S^{1}(t)$, i.e.

$$
\mathcal{M}_{x}^{1}\left(T_{\mid\left(\alpha \cdot S^{1}\right)}(t)\right)=\mathcal{M}_{x}^{1}\left(T_{\mid\left(S^{1}\right)}(t)\right)=\rho+\varepsilon .
$$


Thus we can assume that our curve $S^{1}(t)$, which satisfies (4.5.2), lies in the ball $B(0, \delta)$. By our choice of $\delta$ ( see (4.5.1)), we get from (4.5.2)

$$
\rho+\frac{3}{4} \varepsilon \leq \mathcal{M}^{1}\left(\operatorname{Exp}\left(S^{1}(t)\right)\right) \leq \rho+\frac{5}{4} \varepsilon,
$$

for all $t$. This curve $\operatorname{Exp}\left(S^{1}(t)\right)$ is an immersed curve.

Now let us to continue the proof of Proposition 4.4.a. We denote by $S^{1}(t)$ the embedded curve in Lemma 4.5. Next by choosing a tubular neighborhood of $S^{1}(t)$ we can get a (small, thin) oriented embedded solid torus $T^{3}(t, s, r)=S^{1}(t) \times S^{1}(s) \times[0, R]$ in $M^{m}$ such that our embedded curve is exactly the mean curve $S^{1}(t) \times\{0\} \times\{0\}$ on the solid torus. We can choose this torus $T^{3}$ so thin, such that for all $s, t, r$ we have

$$
\mathcal{M}^{1}\left(T_{r}^{2}(t, s)\right) \geq \rho+\frac{\varepsilon}{4}
$$

Using (4.5.4) we choose a smooth unit vector field $V(t, s)$ on the torus $T^{3}(t, s, r)$ which is tangential to each torus $T_{r}^{2}(t, s)$ such that $T(V, V, V)=\rho$. The integral curve of this vector field is either a circle or an curve of infinite length. If there exists an integral curve of infinite length, then this curve is our desired curve for the Proposition 4.5. Assume now that all the integral curves are circles. Then there exist an embedding $S^{1}(t) \times[0, \mu] \times[0, \mu]$ such that for all $(s, r) \in[0, \mu] \times[0, \mu]$ the circle $S^{1}(t) \times\{s\} \times\{r\}$ is an integral curve of $V$. Now we perturb $V$ in a neighborhood $[0, \alpha] \times[0, \mu] \times[0, \alpha]$ with a very small $\alpha$ such that the perturbed unit vector field $V^{\prime}$ satisfies $T\left(V^{\prime}, V^{\prime}, V^{\prime}\right)=\rho$ and the integral curve of vector field $V^{\prime}$ is not any more periodic. This completes the proof of the first part in Proposition 4.4.

Using the same argument we can prove the second part b) of Proposition 4.4. First we get the existence of an embedded curve $S^{1}(t)$ of arbitrary length on $M$ such that $\mathcal{M}^{1}\left(T_{\mid S^{1}}(t)\right) \geq \rho+(1 / 4) \varepsilon$. Now we consider a torus tubular neighborhood of this curve in $M$ and apply the same argument in the first part, namely we get on each torus $T^{2}(t, s)$ an integral curve whose unit tangential vector $V=(\partial / \partial t) S^{1}(t ; s, r)$ satisfies the condition:

$$
T(V, V, V)=\rho .
$$

If there exists an infinite integral curve, then we are done. If not, that means all integral curve are circles, then we apply the perturbation method in the proof of the first part and get our desired curve.

\section{Existence Of isostatistical EMBeddings into $C a p^{N}$.}

Main Theorem. Any compact smooth ( $C^{1}$ resp.) statistical manifold $(M, g, T)$ can be immersed into the statistical manifold $\left(\mathrm{Cap}_{+}^{N}, g^{F}, T^{A-C}\right)$ for some finite number $N$. Hence any statistical manifold is a statistical model.

We first deduce our Main Theorem from Theorem 5.1 and Theorem 5.5. 
5.1. Theorem. Let $\left(M^{m}, g, T\right)$ be a compact smooth ( $C^{1}$ resp.) statistical manifold. Then there exist numbers $N \in \mathbb{N}^{+}$and $A \geq 0$ as well as a smooth $\left(C^{1}\right.$ resp. $)$ embedding $f:\left(M^{m}, g, T\right) \rightarrow\left(\mathbb{R}^{N}, g_{0}, A \cdot T_{0}\right)$ such that $f^{*}\left(g_{0}\right)=g$ and $f^{*}\left(A \cdot T_{0}\right)=T$.

Our proof of Theorem 5.1 uses the Nash embedding theorem, the Gromov embedding theorem and an algebraic trick. The existence of monotone invariants prevents us extend Theorem 5.1 for non-compact case (in contrast to the Riemannian case.)

5.2. The Nash embedding theorem. [Nash1954, Nash1956] Any smooth ( $C^{1}$ resp.) -Riemannian manifold $\left(M^{n}, g\right)$ can be isometrically embedded into $\left(\mathbb{R}^{N}, g_{0}\right)$ for some $N$ depending on $M^{n}$.

We denote by $T_{0}$ the "standard" 3 -tensor on $\mathbb{R}^{n}$ :

$$
T_{0}=\sum_{i=1}^{n} d x_{i}^{3} .
$$

5.3. The Gromov immersion theorem. [Gromov1986, 2.4.9.3' and 3.1.4] Suppose that $M^{m}$ is given with a smooth ( $C^{1}$ resp.) symmetric 3form $T$. Then there exists an embedding $f: M^{m} \rightarrow \mathbb{R}^{N_{1}(m)}$ with $N_{1}(m)=$ $3\left(n+\left(\begin{array}{l}n+1 \\ 2\end{array}\right)+\left(\begin{array}{l}n+2 \\ 3\end{array}\right)\right)$ such that $f^{*}\left(T_{0}\right)=T$.

Proof of Theorem 5.1. First we shall take an immersion $f_{1}:\left(M^{m}, g, T\right) \rightarrow$ $\left(\mathbb{R}^{N_{1}(m)}, g_{0}, T_{0}\right)$ such that

$$
f_{1}^{*}\left(T_{0}\right)=T
$$

The existence of $f_{1}$ follows from the Gromov immersion theorem.

Then we choose a positive number $A^{-1}$ such that

$$
g-A^{-1}\left(f_{1}^{*}\left(g_{0}\right)\right)=g_{1}
$$

is a Riemannian metric on $M$, i.e. $g_{1}$ is a positive symmetric bi-linear form. Such a number $A$ exists, since $M$ is compact.

Now we shall choose an isometric immersion $f_{2}:\left(M^{m}, g_{1}\right) \rightarrow\left(\mathbb{R}^{N}, g_{0}\right)$. The existence of $f_{2}$ follows from the Nash isometric immersion theorem.

5.4. Lemma. There is a linear isometric embedding $L_{m+1}: \mathbb{R}^{m+1} \rightarrow$ $\mathbb{R}^{2 m+2}$ such that $L_{n+1}\left(T_{0}\right)=0$.

Proof. We put

$$
L_{m+1}\left(x_{1}, \cdots, x_{m+1}\right)=\left(f^{1}\left(x_{1}\right), \cdots, f^{m+1}\left(x_{m+1}\right)\right)
$$

where $f^{i}$ embeds the line $\left(\mathbb{R},\left(d x^{i}\right)^{2}, 0\right)$ into $\left(\mathbb{R}^{2},\left(d x^{2 i-1}\right)^{2}+\left(d x^{2 i}\right)^{2},\left(d x^{2 i-1}\right)^{3}+\right.$ $\left.\left(d x^{2 i}\right)^{3}\right)$ :

$$
f^{i}\left(x_{i}\right)=\frac{1}{\sqrt{2}}\left(x_{2 i-1}-x_{2 i}\right) .
$$

Clearly, $L_{m+1}$ is the required embedding. 
Completion of the proof of Theorem 5.1. Finally we take an embedding

$$
f_{3}: M^{m} \rightarrow \mathbb{R}^{(m+1)(m+2)+m} \oplus \mathbb{R}^{2 m+2}
$$

as follows.

$$
f_{3}(x)=A^{-1} \cdot f_{1}(x) \oplus\left(L_{n+1} \circ f_{2}\right) .
$$

Since $f_{2}$ is an embedding, $f_{3}$ is the required embedding map for Theorem 5.1 .

5.5. Theorem. Suppose that $C$ is a compact subset in $C a p_{+}^{4 n}$. Then any bounded domain $D$ in a linear statistical manifold $\left(\mathbb{R}^{n}, g_{0}, A \cdot T_{0}\right)$ can be realized as an immersed statistical submanifold of $\left(\mathrm{Cap}_{+}^{4 n}, g^{F}, T^{A-C}\right)$.

Set

$$
T^{*}:=\sum_{i=1}^{n} \frac{2 d x_{i}^{3}}{x_{i}} .
$$

We denote by $S_{r,+}^{n}$ the positive sector of the sphere of radius $r$ centered at the origin in $\mathbb{R}^{n+1}$.

Proof of Theorem 5.5. We choose a very large positive number

$$
\bar{A}=\bar{A}(n, A)
$$

to be specified in Lemma 5.1 later. First, $\bar{A}$ in (5.1) is required to be so large such that there exists a number $1<\lambda<2$ satisfying the following equation

$$
\lambda^{2}+\frac{3 n}{(2 \bar{A})^{2}}=4
$$

Equation (5.2) implies that $\left(\lambda,(2 \bar{A})^{-1},(2 \bar{A})^{-1},(2 \bar{A})^{-1}\right) \in \mathbb{R}^{4}$ is a point in $S_{2 / \sqrt{n},+}^{3}$. Hence there exists a positive number $r(\bar{A})$ such that for all $0<$ $r \leq r(\bar{A})$ the ball $U(\bar{A}, r)$ of radius $r$ in the sphere $S_{2 / \sqrt{n}}^{3}$ that is centered at the point $\left(\lambda,(2 \bar{A})^{-1},(2 \bar{A})^{-1},(2 \bar{A})^{-1}\right)$ belongs also to the positive quadrant $S_{2 / \sqrt{n},+}^{3}$. Hence $U(\bar{A}, r) \times{ }_{\mathrm{n} \text { times }} U(\bar{A}, r)$ is a subset in $S_{2,+}^{4 n-1} \subset \mathbb{R}^{4 n}$.

Next, we note that Theorem 5.5 is a consequence of the following

Lemma 5.1. For given positive numbers $R>0$ and $A \geq 0$ there exist a positive number $\bar{A}$, satisfying (5.2) and depending only on $n$ and $A, a$ positive number $r<r(\bar{A})$ and an isostatistical immersion $h$ from the bounded domain $[0, R] \times{ }_{n \text { times }}[0, R] \subset\left(\mathbb{R}^{n}, g_{0}, A \cdot T_{0}\right)$ into $\left(\mathrm{Cap}_{+}^{4 N}, g^{F}, T^{A-C}\right)$ such that $h:[0, R] \times{ }_{n \text { times }}[0, R] \subset U(\bar{A}, r) \times{ }_{n}$ times $U(\bar{A}, r)$.

Proof. Set

$$
T^{*}:=\sum_{i=1}^{n} \frac{2 d x_{i}^{3}}{x_{i}} .
$$

Since $\left(U(\bar{A}, r),\left.\left(g_{0}\right)\right|_{U(\bar{A}, r)},\left.T^{*}\right|_{U(\bar{A}, r)}\right)$ is a statistical submanifold of $\left(\mathbb{R}_{+}^{4}, g_{0}, T^{*}\right)$, the direct product

$$
\left(U(\bar{A}, r) \times_{\mathrm{n} \text { times }} U(\bar{A}, r),\left.\oplus_{i=1}^{n}\left(g_{0}\right)\right|_{U(\bar{A}, r)},\left.\oplus_{i=1}^{n} T^{*}\right|_{U(\bar{A}, r)}\right)
$$


is a statistical submanifold of $\left(\mathbb{R}_{+}^{4 n}, g_{0}, T^{*}\right)$. Since $\left(\operatorname{Cap}_{+}^{N}, g^{F}, T^{A-C}\right)$ is a statistical submanifold of $\left(R_{+}^{N}, g_{0}, T^{*}\right)$, we conclude that

$$
\left(U(\bar{A}, r) \times{ }_{\mathrm{n} \text { times }} U(\bar{A}, r),\left.\oplus_{i=1}^{n}\left(g_{0}\right)\right|_{U(\bar{A}, r)},\left.\oplus_{i=1}^{n} T^{*}\right|_{U(\bar{A}, r)}\right)
$$

is a statistical submanifold of $\left(\operatorname{Cap}_{+}^{4 N}, g^{F}, T^{A-C}\right)$. Hence, to prove Lemma 5.1, it suffices to show that there are positive numbers $\bar{A}=\bar{A}(n, A), r<r(\bar{A})$ and an isostatistical immersion $f:\left([0, R], d x^{2}, A \cdot d x^{3}\right) \rightarrow\left(U(\bar{A}, r),\left.\left(g_{0}\right)\right|_{U(\bar{A}, r)},\left.T^{*}\right|_{U(\bar{A}, r)}\right)$. On $U(\bar{A}, r)$ we consider the distribution $D(\rho)$ defined by

$$
D_{x}(\rho):=\left\{v \in T_{x} U(\bar{A}, r):|v|_{g_{0}}=1, T^{*}(v, v, v)=\rho\right\}
$$

for any given $\rho>0$. Clearly the existence of an isostatistical immersion $f:\left([0, \mathbb{R}], d x^{2}, A \cdot d x^{3}\right) \rightarrow\left(U(\bar{A}, r),\left.\left(g_{0}\right)\right|_{U(\bar{A}, r)},\left.T^{*}\right|_{U(A, r)}\right)$ is equivalent to the existence of an integral curve with the length $R$ of the distribution $D(A)$ on $U(\bar{A}, r)$.

Now we are going to prove the following

Lemma 5.2. There exist a positive number $\bar{A}=\bar{A}(n, A)$ and an embedded torus $T^{2}$ in $U(\bar{A}, r)$ which is provided with a unit vector field $V$ on $T^{2}$ such that $T^{*}(V, V, V)=A$.

Proof of Lemma 5.2, Let us denote

$$
x_{0}:=\left(\lambda,(2 \bar{A})^{-1},(2 \bar{A})^{-1},(2 \bar{A})^{-1}\right) \in S^{3}(2 / \sqrt{n})
$$

with $\lambda$ defined by (5.2). We shall need the following

Lemma 5.3. There exists a positive number $\bar{A}=\bar{A}(n, A)$ such that the following assertion holds. Let $H$ be any 2-dimensional subspace in $T_{x_{0}} U(\bar{A}, r) \subset$ $\mathbb{R}^{4}$. Then there exists a unit vector $w \in H$ such that $T^{*}(w, w, w) \geq \sqrt{2} A$.

Proof of Lemma 5.3. Denote by $\vec{x}_{0}$ the vector in $\mathbb{R}^{4}$ with the same coordinates as those of the point $x_{0}$. For any given $H$ as in Lemma 5.3 there exists a unit vector $\vec{h}$ in $\mathbb{R}^{4}$, which is not co-linear with $\vec{x}_{0}$ and which is orthogonal to $H$, such that a vector $w \in \mathbb{R}^{4}$ belongs to $H$ if and only if $w$ is a solution to following two linear equations:

$$
\begin{gathered}
\left\langle w, \vec{x}_{0}\right\rangle=0, \\
\langle w, \vec{h}\rangle=0 .
\end{gathered}
$$

Adding a multiple of $\vec{x}_{0}$ to $\vec{h}$ if necessary, and taking the normalization, we can assume that

$$
\vec{h}=\left(0=h_{1}, h_{2}, h_{3}, h_{4}\right) \text { and } \sum_{i} h_{i}^{2}=1 .
$$


Case 1. Suppose that not all the coordinates $h_{i}$ of $\vec{h}$ are of the same sign, so w.l.o.g. we assume that $h_{1}=0, h_{2} \leq 0, h_{3}>0$. We put

$$
\begin{array}{r}
k_{2}:=\frac{-h_{2}}{\sqrt{\left(h_{2}\right)^{2}+\left(h_{3}\right)^{2}}}, k_{3}:=\frac{h_{3}}{\sqrt{\left(h_{2}\right)^{2}+\left(h_{3}\right)^{2}}}, \\
w:=\left(w_{1}, w_{2}=\left(1-\varepsilon_{2}\right) k_{3}, w_{3}=\left(1-\varepsilon_{2}\right) k_{2}, 0=w_{4}\right) \in \mathbb{R}^{4} .
\end{array}
$$

Obviously, for any choice of $w_{1}$ and $\varepsilon_{2}$ the equation (5.4) for $w$ is satisfied. Now we choose $w_{1}, \varepsilon_{2}$ to be solutions of the following equations

$$
\begin{gathered}
\lambda \cdot w_{1}+\left(1-\varepsilon_{2}\right) \cdot(2 \bar{A})^{-1} \cdot\left(k_{2}+k_{3}\right)=0, \\
w_{1}^{2}=\left(2 \varepsilon_{2}-\varepsilon_{2}^{2}\right) .
\end{gathered}
$$

Note that (5.6) is equivalent to (5.3) and (5.7) normalizes $w$. From (5.6) we get

$$
w_{1}=-\frac{\left(1-\varepsilon_{2}\right)\left(k_{2}+k_{3}\right)}{\lambda \cdot 2 \bar{A}} .
$$

Substituting the value of $w_{1}$ into (5.7), we get

$$
\left(\frac{\left(k_{2}+k_{3}\right)^{2}}{(\lambda \cdot 2 \bar{A})^{2}}+1\right) \varepsilon_{2}^{2}-\left(2+\frac{2\left(k_{2}+k_{3}\right)^{2}}{(\lambda \cdot 2 \bar{A})^{2}}\right) \varepsilon_{2}+\left(\frac{k_{2}+k_{3}}{\lambda \cdot 2 \bar{A}}\right)^{2}=0,
$$

which we simplify as follows:

$$
\varepsilon_{2}^{2}-2 \varepsilon_{2}+\frac{\left(k_{2}+k_{3}\right)^{2}}{\left(k_{2}+k_{3}\right)^{2}+4 \lambda^{2} \bar{A}^{2}}=0 .
$$

Clearly, the following choice of $\varepsilon_{2}$ is a solution to (5.9)

$$
\varepsilon_{2}=1-\frac{2 \lambda \bar{A}}{\sqrt{\left(k_{2}+k_{3}\right)^{2}+4 \lambda^{2} \bar{A}^{2}}} .
$$

By our assumption on $h_{2}$ and $h_{2}$, we have $0 \leq k_{2}, k_{3} \leq 1$. Since $1<\lambda<2$ by (5.2), we conclude that when $\bar{A}$ goes to infinity, the value $\varepsilon_{2}$ goes to zero. Hence there exists a number $N_{1}>0$ such that if $\bar{A}>N_{1}$ then

$$
\varepsilon_{2}>0 \text { and }\left(1-\varepsilon_{2}\right)^{2} \geq \frac{3}{4}
$$

We shall show that for $\varepsilon_{2}$ in (5.10) that also satisfies (5.11) if $\bar{A}$ is sufficiently large, and for $w_{1}$ defined by (5.8), the vector $w$ defined by (5.5) satisfies the required condition of Lemma $[5.3)$. Since $x_{0}=\left(\lambda,(2 \bar{A})^{-1},(2 \bar{A})^{-1},(2 \bar{A})^{-1}\right)$ we have

$$
T_{x_{0}}^{*}(w, w, w)=\frac{2 w_{1}^{3}}{\lambda}+(4 \bar{A})\left(w_{2}^{3}+w_{3}^{3}\right) .
$$

Now assume that $\bar{A}>N_{1}$. Noting that $\varepsilon_{2}$ is positive and close to zero, and using $k_{2} \geq 0, k_{3} \geq 0$, we obtain from (5.5)

$$
w_{2} \geq 0, w_{3} \geq 0 \text {. }
$$


Since $0<\varepsilon<1,0<k_{2}+k_{3}<2$, and $\lambda, \bar{A}$ are positive, we obtain from (5.8)

$$
w_{1}<0 \text { and }\left|w_{1}\right|<\frac{1}{\lambda \bar{A}} .
$$

Taking into account (5.5) and (5.11), we obtain

$$
w_{2}^{2}+w_{3}^{2}=\left(1-\varepsilon_{2}\right)^{2} \geq \frac{3}{4} .
$$

Using (5.14), we obtain from (5.12)

$$
T_{x_{0}}^{*}(w, w, w) \geq \frac{-2}{\lambda^{4} \bar{A}^{3}}+(4 \bar{A}) \cdot\left(w_{2}^{3}+w_{3}^{3}\right) .
$$

Observing that the function $x^{3 / 2}+(c-x)^{3 / 2}$ is convex on interval $[0, c]$ for any $c>0$, using (5.13) and (5.15), we obtain from (5.16)

$$
\left.T_{x_{0}}^{*}(w, w, w) \geq \frac{-2}{\lambda^{4} \bar{A}^{3}}+(4 \bar{A}) \cdot 2\left(\frac{\sqrt{3}}{\sqrt{2}}\right)^{3}\right)=\frac{-2}{\lambda^{4} \bar{A}^{3}}+8\left(\sqrt{\frac{3}{2}}\right)^{3} \bar{A} .
$$

Increasing $\bar{A}$ if necessary, noting that $1<\lambda=\lambda(A)$, equation (5.17) implies that there exists a large positive number $\bar{A}(n, A)$ depending only on $n$ and $A$ such that any subspace $H$ defined by the equations (5.3) and (5.4), where $h$ is in Case 1, contains a unit vector $w$ that satisfies the condition in Lemma 5.3. i.e. the RHS of (5.17) is larger than $\sqrt{2} A$.

Case 2. W.l.o.g. we assume that $h_{2} \geq h_{3} \geq h_{4}>0$ and therefore we have

$$
\alpha:=\frac{h_{2}+h_{3}}{h_{4}} \geq 2 .
$$

We shall search the required vector $w$ for Lemma 5.3 in the following form

$$
w:=\left(w_{1}, w_{2}=-\left(1-\varepsilon_{2}\right), w_{3}=-\left(1-\varepsilon_{2}\right), w_{4}=\alpha\left(1-\varepsilon_{2}\right)\right) .
$$

The equations (5.19) and (5.18) ensure that $\langle w, \vec{h}\rangle=0$ for any choice of parameters $\left(w_{1}, \varepsilon_{2}\right)$ of $w$ in (5.19). Next we require that the parameters $\left(w_{1}, \varepsilon_{2}\right)$ of $w$ satisfy the following two equations

$$
\begin{gathered}
\lambda \cdot w_{1}+\frac{\left(1-\varepsilon_{2}\right)(\alpha-2)}{2 \bar{A}}=0, \\
w_{1}^{2}+\left(1-\varepsilon_{2}\right)^{2}\left(2+\alpha^{2}\right)=1 .
\end{gathered}
$$

Note that (5.20) is equivalent to (5.3) and (5.21) normalizes $w$. From (5.20) we express $w_{1}$ in terms of $\varepsilon_{2}$ as follows

$$
w_{1}=-\frac{\left(1-\varepsilon_{2}\right)(\alpha-2)}{\lambda 2 \bar{A}} .
$$

Set

$$
B:=\left(2+\alpha^{2}\right)+\frac{(\alpha-2)^{2}}{4 \lambda^{2} \bar{A}^{2}} .
$$


Plugging (5.22) into (5.21) and using (5.23), we obtain the following equation for $\varepsilon_{2}$

$$
\left(1-\varepsilon_{2}\right)^{2} B-1=0
$$

which is equivalent to the following equation

$$
\left(1-\varepsilon_{2}\right)^{2}=\frac{1}{B} \text {. }
$$

Since $\alpha \geq 2$ by (5.18), from (5.23) we have $B>0$. Clearly

$$
\varepsilon_{2}:=1-\frac{1}{\sqrt{B}}
$$

is a solution to (5.24).

Since $\alpha \geq 2$ and $\varepsilon_{2} \leq 1$ by (5.25), we obtain from (5.22) that $w_{1} \leq 0$. Taking into account $1<\lambda, \bar{A}>0$, we derive from (5.22) and (5.25) the following estimates

$$
\begin{gathered}
T_{x_{0}}^{*}(w, w, w)=\frac{2 w_{1}^{3}}{\lambda}+(4 \bar{A})\left(1-\varepsilon_{2}\right)^{3}\left(\alpha^{3}-2\right) \\
>2 w_{1}^{3}+(4 \bar{A})\left(\alpha^{3}-2\right)\left(1-\varepsilon_{2}\right)^{3} \\
=\frac{(\alpha-2)^{3}}{4 \bar{A}^{3}(\sqrt{B})^{3}}+4 \bar{A} \frac{\left(\alpha^{3}-2\right)}{(\sqrt{B})^{3}} \\
\geq-\frac{\alpha^{3}-2}{4 \bar{A}^{3}(\sqrt{B})^{3}}+4 \bar{A} \frac{\left(\alpha^{3}-2\right)}{(\sqrt{B})^{3}}(\text { since } \alpha \geq 2) \\
=\frac{\alpha^{3}-2}{(\sqrt{B})^{3}}\left(-\frac{1}{4 \bar{A}^{3}}+4 \bar{A}\right) .
\end{gathered}
$$

Lemma 5.4. There exists a large number $\bar{A}=\bar{A}(n, A)$ depending only on $n$ such that for all choice of $\alpha \geq 2$ we have

$$
\frac{\left(\alpha^{3}-2\right)}{(\sqrt{B})^{3}} \geq \frac{1}{10^{2}} .
$$

Proof. To prove Lemma 5.4 it suffices to show that for $\alpha \geq 2$ we have

$$
10^{4}\left(\alpha^{3}-2\right)^{2} \geq B^{3} \text {. }
$$

Clearly there exists a positive number $N_{2}$ such that if $\bar{A}>N_{2}$, then by (5.23), we have

$$
B<\frac{3}{2}\left(2+\alpha^{2}\right)
$$

for any $\alpha \geq 2$. Hence (5.27) is a consequence of the following relation

$$
10^{4}\left(\alpha^{3}-2\right)^{2} \geq\left[\frac{3}{2}\left(2+\alpha^{2}\right)\right]^{3},
$$

which we shall establish now. To prove (5.29) it suffices to show that

$$
10^{3}\left(\alpha^{3}-2\right)^{2} \geq\left(2+\alpha^{2}\right)^{3} .
$$


The inequality (5.30) is equivalent to the following

$$
999 \alpha^{6}-6 \alpha^{4}-4000 \alpha^{3}-12 \alpha^{2}+3992 \geq 0 .
$$

Since $\alpha \geq 2$ it follows that $\alpha^{3} \geq 8$ and hence

$$
999 \alpha^{6}-4000 \alpha^{3}=499 \alpha^{6}+500 \alpha^{3}\left(\alpha^{3}-8\right) \geq 499 \alpha^{6} .
$$

Using $2 \alpha^{6} \geq 6 \alpha^{4}$, we obtain

$$
499 \alpha^{6}-6 \alpha^{4} \geq 497 \alpha^{6}
$$

Using $a^{4} \geq 16$, we obtain

$$
497 \alpha^{6}-12 \alpha^{2}=496 \alpha^{6}+\alpha^{2}\left(\alpha^{4}-12\right)>496 \alpha^{6} .
$$

From (5.32), (5.33), (5.34) we obtain

$$
999 \alpha^{6}-6 \alpha^{4}-4000 \alpha^{3}-12 \alpha^{2}+3992 \geq 496 \alpha^{6}+3992>0 .
$$

This proves (5.30) and hence completes the proof of Lemma 5.4.

Lemma 5.4 implies that when $\bar{A}=\bar{A}(A, n)$ is sufficiently large, the RHS of (5.26) is larger than $\sqrt{2} A$. This proves the existence of $\bar{A}$, which depends only on $n$ and $A$, for Case 2 .

This completes the proof of Lemma 5.3 .

From Lemma 5.3 we obtain immediately the following.

Corollary 5.5. The exists a small neighborhood $U_{1} \ni x_{0}$ in $\bar{U}(\bar{A}, r)$ such that the following statement holds. For any $x \in U_{1}$ and any two-dimensional subspace $H \subset T_{x} U_{1}$ we have

$$
\max \left\{T^{*}(v, v, v) \mid v \in H \text { and }|v|_{g_{0}}=1\right\} \geq \frac{5}{4} A .
$$

Completion of the proof of Lemma 5.2. Let $\bar{A}=\bar{A}(n, A)$ satisfy the condition of Lemma 5.3. Now we choose a small embedded torus $T^{2}$ in $U_{1} \subset U(\bar{A}, r)$. By Corollary [5.5, for all $x \in T^{2}$ we have

$$
\max \left\{T^{*}(v, v, v) \mid v \in T_{x} T^{2} \text { and }|v|_{g_{0}}=1\right\} \geq \frac{5}{4} A .
$$

Denote by $T_{1} T^{2}$ the bundle of the unit tangent vectors of $T^{2}$. Since $T^{2}=\mathbb{R}^{2} / \mathbb{Z}^{2}$ is parallelizable, we have $T_{1} T^{2}=T^{2} \times S^{1}$. Thus the existence of a vector field $V$ required in Lemma 5.2 is equivalent to the existence of a function $T^{2} \rightarrow S^{1}$ satisfying the condition of Lemma 5.2, Next we claim that there exists a unit vector field $W$ on $T^{2}$ such that $T^{*}(W, W, W)=0$. First we choose some orientation for $T^{2}$, that induces an orientation on $T_{1} T^{2}$ and hence on the circle $S^{1}$. Take an arbitrary unit vector field $W^{\prime}$ on $T^{2}$, equivalently we pick a function $W^{\prime}: T^{2} \rightarrow S^{1}$. Now we consider the fiber bundle $F$ over $T^{2}$ whose fiber over $x \in T^{2}$ consists of the interval $\left[W^{\prime},-W^{\prime}\right]$ defined by the chosen orientation on the circle of unit vectors in $T_{x} S^{2}$. Since $T^{*}\left(W^{\prime}, W^{\prime}, W^{\prime}\right)=-T^{*}(W, W, W)$, for each $x \in T^{2}$ there exists a value $W$ on $F(x)$ such that $T^{*}(W, W, W)=0$ and $W$ is closest to $W^{\prime}$. Using $W$ we 
identify the circle $S^{1}$ with the interval $[0,1)$. The existence of $W$ implies that the existence of a function $V: T^{2} \rightarrow[0,1)$, regarded as a unit vector field $V$ on $T^{2}$, that satisfies the condition of Lemma 5.2 is equivalent to the existence of a function $f: T^{2} \rightarrow[0,1)$ satisfying the same condition. Now let $V(x)$ be the smallest value of unit vector $V(x) \in[0,1) \subset S^{1}\left(T_{x} T^{2}\right)$ such that

$$
T^{*}(V(x), V(x), V(x))=A
$$

for each $x \in T^{2}$. The existence of $V(x)$ follows from (5.36). This completes the proof of Lemma 5.2 .

As we have noted, Lemma 5.2 implies Lemma 5.1 .

This finishes the proof of Theorem 5.5.

Proof of Main Theorem. The existence of an isostatistical immersion of a compact statistical manifold $(M, g, T)$ into $\left(\mathrm{Cap}_{+}^{N}, g^{F}, T^{A-C}\right)$ for some finite $N$ follows from Theorem 5.1 and Theorem 5.5.

Theorem 5.6. Any smooth ( $C^{1}$ resp.) compact statistical manifold $\left(M^{n}, g, T\right)$ admits an isostatistical embedding into the statistical manifold $\left(\mathcal{P}_{+}([N]), g^{F}, T^{A-C}\right)$ for some finite number $N$.

Proof. To prove Theorem 5.6 we repeat the proof of Main Theorem, replacing the Nash immersion theorem by the Nash embedding theorem. First we observe that our immersion $f_{3}$ constructed in the proof of Lemma 5.4 is an embedding, if $f_{2}$ is an isometric embedding. The existence of an isometric embedding $f_{2}$ is ensured by the Nash theorem. Hence, if $M^{n}$ is compact, to prove the existence of an isostatistical embedding of $\left(M^{n}, g, T\right)$ into $\left(\mathcal{P}_{+}([N]), g^{F}, T^{A-C}\right)$ it suffices to prove the strengthened version of Theorem 5.5, where the existence of an isostatistical immersion is replaced by the existence of an isostatistical embedding.

Recall that the proof of Theorem 5.5 is reduced to the proof of the existence of an isostatistical immersion of a bounded statistical interval $\left([0, R], d t^{2}, A \cdot d t^{3}\right)$ into a torus $T^{2}$ of a small domain in $\left(S_{2 / \sqrt{n},+}^{3}, g_{0}, T^{*}\right)$, see the proof of Lemma 5.1. Here for simplicity of notation, we abbreviate the restriction of $T^{*}$ to the sphere in consideration as $T^{*}$.

The statistical immersion produced with the help of Lemma 5.2 will be an embedding if not all the integral curves of the distribution $D(A)$ on the torus $T^{2}$ are closed curves. Now we shall search for an isostatistical embedding of $\left([0, R], d t^{2}, A \cdot d t^{3}\right)$ into a torus $T^{2} \times T^{2}$ of a small domain in $\left(S_{1 / \sqrt{n},+}^{3}, g_{0}, T^{*}\right) \times\left(S_{1 / \sqrt{n},+}^{3}, g_{0}, T^{*}\right) \subset\left(\mathbb{R}^{8}, g_{0}, T^{*}\right)$. Since $T^{4}$ is parallelizable, repeating the argument at the end of the proof of Lemma 5.1, we choose a distribution $D(A) \subset T T^{4}$ such that $D(A)=T^{4} \times S^{2}$ and

$$
D_{x} A=\left\{\left.v \in T_{x} T^{4}|| v\right|_{g_{0}}=1, \text { and } T^{*}(v, v, v)=A\right\} .
$$


Now assume that the integral curves of $D(A)$ that lie on the first factor $T^{2} \times y$ for all $y \in S_{1 / \sqrt{n},+}^{3}$ are closed. Since $T^{2}$ is compact, there is a positive number $p_{1}$ such that the periods of these integral curves are at least $p_{1}$.

Now let us consider the following integral curve $\gamma(t)$ of $D(A)$ on $T^{4}$. The curve $\gamma(t)$ begins at a point $(0,0,0,0) \in T^{4}$. Here we identify $T^{1}$ with $[0,1] /(0=1)$. The integral curve lies on $T^{2} \times(0,0)$ until it approaches $(0,0,0,0)$ again. Since $D_{x}(A)=S^{2}$, we can slightly modify the direction of $\gamma(t)$ and let it leave the torus $T^{2} \times(0,0)$ and after a very short time $\gamma(t)$ must stay on the torus $T^{2} \times(\varepsilon, \varepsilon)$ where $\varepsilon$ is sufficiently small. W.l.o.g. we assume that the period of any closed curve of the distribution $D(A) \cap T\left(T^{2} \times\right.$ $(\varepsilon, \varepsilon))$ is at least $p_{1}$. Repeating this procedure, since $R$ and $p_{1}$ are finite, we produce an embedding of $\left([0, R], d t^{2}, A \cdot d t^{3}\right)$ into $T^{4} \subset\left(S_{1 / \sqrt{n},+}^{3}, g_{0}, T^{*}\right) \times$ $\left(S_{1 / \sqrt{n},+}^{3}, g_{0}, T^{*}\right)$.

This completes the proof of Theorem 5.6 .

\section{REFERENCES}

[A-N2000] S. Amari And H. NAGAOKA, Methods of Information Geometry, Trans. of Math. Monograph (2000).

[Amari1997] S. AmARI, Information geometry, Contemporary Math. vol 203, 81-95, (1997).

[Ay2002] N. Ay, An information-geometric approach to a theory of pragmatic structuring, Ann. of Prob., v.30, N. 1, 416-436 (2002).

[Chentsov1972] N.N. Chentsov, Statistical decision rules and optimal inference, AMS., (1982), (originally published in Russian, Nauka, Moscow , 1972).

[Gromov1986] M. Gromov, Partial Differential Relations, Springer-Verlag, (1986).

[Jost2005] J. JosT, Information geometry, lecture at MPI MIS 2005.

[Lauritzen1987] S. LAURITzen, Statistical manifolds, In : Differential geometry in Statistical Inference, IMS Lecture Notes, Monograph Serie 10., Inst. of Math. Stat. Hayward, California, (1987).

[Matsumoto1993] T. Matumoto, Any statistical manifold has a contrast function - On the $C^{3}$-functions taking the minimum at the diagonal of the product manifold, Hiroshima Math. J., 23 , 327-332, (1993).

[M-C1990] E.A. Morozova AND N.N. Chentsov, Markov invariant geometry on manifolds of states (in Russian), Itogi Nauki i Techniki 36, 69-102, (1990).

[Nash1954] J. NAsh, $C^{1}$-isometric imbeddings, Ann. Math. 60, 383-396, (1954).

[Nash1956] J. NAsh, The imbedding problem for Riemannian manifolds, Ann. of Math., 63, 20-64, (1956).

[O-V1988)] E.B.VinberG, A.L. OnishchiK, Seminars on Lie groups and algebraic groups, (in Russian) Nauka, Moscow, (1988).

[Rao1945] C. R. RAO, Information and accuracy attainable in the estimation of statistical parameters, Bull. of the Calcutta Math. Soc. 37, 89-91 (1945).

[Rao1987] C. R RAO, Differential metrics in probability spaces, In : Differential geometry in Statistical Inference, IMS Lecture Notes, Mongraph Serie 10., Inst. of Math. Stat. Hayward, California, (1987). 